ures are adopted. These might include the screening and treatment of pregnant women and the further development of the tetanus toxoid GBS polysaccharide conjugate vaccines, which should be ready for human trials later this year.

From Infectious D iseases in Children. June 1991;4:1.

\section{Tuberculosis Takes a Turn for the Worse}

For decades, tuberculosis (TB) has been declining an average of 5\% each year; in 1985, incidence began to level off, and then in 1989, it increased by about 5\%. Preliminary data suggest that U.S. cases increased by $9 \%$ in 1990 .

"This is a dramatic shift, unprecedented in the history of this country," said Dixie Snider Jr., MD, Centers for Disease Control (CDC), Atlanta, Georgia.

To deter the rise of this disease, the American Thoracic Society and the CDC collaborated to produce the following preventive therapy guidelines, which they reported at the 1991 meeting of the American College of Physicians.

About $90 \%$ of the current tuberculin cases occur among the 10 million previously infected people in this country "Obviously, unless preventive therapy is applied to reduce this reservoir, hundreds of thousands of new tuberculosis cases and tens of thousands of deaths will occur," said Lee B. Reichman, MD, University of Medicine and Dentistry of New Jersey, Newark, New Jersey.

"(Human immunodeficiency virus) HIV infection is the strongest risk factor for the development of tuberculosis that we've ever seen," he added. Reichman's group showed in a study recently published in The Journal of the American M edical Association that the risk of TB in HIV-infected people is so high that preventative therapy could be prescribed for most groups, except for black women, without TB testing.

Close contacts of people with newly diagnosed infectious TB are candidates for preventive therapy if their skin test reads $5 \mathrm{~mm}$. TB-negative children and adolescents, who have been in close contact with infectious TB also should be treated. People who have an abnormal chest $\mathrm{x}$-ray that shows fibrotic lesions likely to represent healed TB and have a reading of 5 $\mathrm{mm}$ should be treated.

The cut-off point is $10 \mathrm{~mm}$ for intravenous drug users who are HIV-negative and for people with medical conditions that reportedly increase the risk of $\mathrm{TB}$, such as immunosuppression, steroid treatment, gastrectomy, and weight loss."People in these high- risk groups should receive preventive therapy regardless of age," Reichman said.

In the absence of any risk factors, people younger than 35 years of age and the following high-incidence groups should receive preventive therapy if their reaction to a tuberculin test is $10 \mathrm{~mm}$ or more. Highincidence groups include people born in highprevalence countries; medically underserved low income populations, especially blacks, Hispanics, and Native Americans; and residents of long-term care facilities. For people older than 35 years of age, treat those whose skin tests show more than a $15 \mathrm{~mm}$ increase within a two-year period.

According to John B. Bass Jr., South Alabama College of Medicine, Mobile, Alabama, chest x-ray screening for active TB in high-risk groups is a good idea.

'The population determines whether you start out with a skin test or a chest $\mathrm{x}$-ray. For example, the homeless are not likely to return for a skin test reading three days later, then again for a chest x-ray. In this population, it is a good idea to get a chest x-ray. For a population you expect to be immunologically intact and not to have a lot of HIV-positive people with false- negative skin test reactions, etc., a skin test would be the first thing to do," he said.

The usual regimen for preventive therapy is isoniazid, $300 \mathrm{mg}$ maximum per day: $10 \mathrm{mg} / \mathrm{kg} / \mathrm{d}$ for children and $5 \mathrm{mg} / \mathrm{kg}$ for adults for six to 12 months. Isoniazid can be given twice weekly in doses of 20 $\mathrm{mg} / \mathrm{kg}$ to $40 \mathrm{mg} / \mathrm{kg}$ in children or $15 \mathrm{mg} / \mathrm{kg}$ in adults, with a maximum of $900 \mathrm{mg}$. "To ensure compliance in high-risk groups, healthcare personnel should directly observe the therapy," said Reichman.

Oral isoniazid is well tolerated. Mild hepatic dysfunction occurs in $10 \%$ to $20 \%$ of people on isoniazid, but it is seen early in treatment and is usually self-limited, though occasionally progressive. The progressive liver damage is age related and only exists during treatment.

However, Reichman pointed out that avoiding treatment is riskier than possible side effects. Although pyrazinamide and rifampin have not been tested as preventive therapy and they have some toxicity, Bass noted, "In someone who doesn't tolerate isoniazid, I certainly would use rifampin and pyrazinamide for two months or rifampin for four or six months as prophylaxis even though I wasn't absolutely certain it worked."

When patients present with $\mathrm{TB}$, organisms exist in a variety of environments inside the body The majority grow rapidly inside cavities, and these organisms are responsible for the clinical aspects of the illness. Because this population of organisms is large, it is also the most important population to kill quickly 
to prevent the emergence of drug resistance. Inadequate therapy during the first couple of months of treatment will lead to the selection of drug-resistant mutants.

Isoniazid is rapidly bactericidal for Mycobacterium tuberculosis organisms that are in this rapid growth phase.

"Isoniazid is so effective that it will kill $50 \%$ or more of organisms every day or two for the first few days of therapy, and this will have a dramatic effect on the transmissibility of disease," said Bass.

Other organisms grow more slowly in other locations in the body, and because of their slow growth, their location or some unknown effect, prolonged therapy is required.

Rifampin, another excellent anti-TB drug, is not as effective against actively growing organisms, but in experimental conditions is much better than isoniazid at killing slowly growing organisms. The usual dose is $10 \mathrm{mg} / \mathrm{kg} / \mathrm{d}$ to $20 \mathrm{mg} / \mathrm{kg} / \mathrm{d}$ in children or $10 \mathrm{mg} / \mathrm{kg} /$ $\mathrm{d}$ in adults, neither to exceed $600 \mathrm{mg} / \mathrm{d}$.

Pyrazinamide when added to regimens containing both isoniazid and rifampin allows therapy to be shortened to around six months. It works against organisms that are growing slowly or intermittently. Most of the effects of pyrazinamide occur within the first couple of months of therapy, so it need not be given for the entire duration. The dose is $15 \mathrm{mg} / \mathrm{kg} / \mathrm{d}$ to $30 \mathrm{mg} / \mathrm{kg} / \mathrm{d}$ daily in children and adults, with a maximum of $2 \mathrm{~g} / \mathrm{d}$.

Isoniazid and rifampin regimens must be given for nine or ten months to ensure a relapse rate of $2 \%$ or less. "Reducing isoniazid and rifampin regimens below nine months leads to unacceptable relapse rates," said Bass.

However, relapse rates of less than $2 \%$ can be achieved after only six months if pyrazinamide is added to the isoniazid and rifampin regimen. Regimens of INH, rifampin and pyrazinamide for two months followed by an additional four months of isoniazid, and rifampin are at least as effective as the older regimens.

'The major determinant of the outcome of treatment is patient compliance. One cannot simply say, 'Here, take this medication, it's good for you.' The responsibility must be shared by the person delivering care," Bass said.

To treat TB in people with HIV, the current recommendations are to use the standard regimens. One may want to add streptomycin or ethambutol to avoid drug resistance. Therapy should be continued about three months longer than normal. "Try to ensure a period of at least six months of sputum negativity before discontinuing therapy. After successful therapy you do not need to continue medications indefinitely," said Bass.

Bass reported that pregnant women with TB should be treated. The danger of having tuberculosis disease in both the mother and fetus overwhelms any possible complications in drugs, although physicians should consider that streptomycin can induce significant auditory damage in the fetus, and pyrazinamide has not been well studied.

The United States has seen pockets of increased drug resistance, and there are outbreaks of drugresistant disease. In a New York outbreak, 32 cases were resistant to isoniazid and streptomycin. In an outbreak in a Florida hospital, at least 61 cases are resistant to isoniazid, rifampin and often other drugs as well. Thirteen of 40 contacts with these patients became infected, and one of these developed TB pericarditis, Snider reported.

'We had an outbreak in a drug treatment center with only one case, but 15 of 69 contacts in the drug treatment center tested tuberculin positive," he said.

For isoniazid-resistant disease, one option is treatment with rifampin and ethambutol for 12 months. Adding pyrazinamide to this regimen will shorten that duration to nine months. The combination of isoniazid, rifampin, pyrazinamide and either streptomycin or ethambutol given as a six-month regimen, stopping pyrazinamide at the end of the second month and adjusting the regimen for known drug resistance is effective, Bass reported.

"If you're also resistant to rifampin, like those in the Miami outbreak, you're in trouble. Rifampinresistant patients do not respond well to the six-month and nine-month regimens regardless of other resistance patterns," said Bass.

Hope may be on the horizon. The quinolones, the macrolides and the $\beta$-lactams like imipenem may have some effectiveness, but they have not been well studied.

From Infectious D isease N ews. June 1991;4:1. 
